\title{
Engineering the Optical Response of the Novel Plasmonic Binary Nanohole Array
}

Mahdi Javidnasab ( $\square$ Mahdijavidnasab@gmail.com )

University of Tabriz https://orcid.org/0000-0003-1145-5193

Saeid Khesali Azadi

Young Researchers and Elite Club

Majid Ahmadpouri Legha

Young Researchers and Elite Club

Hamid Naghshara

University of Tabriz

\section{Research Article}

Keywords: FDTD, EOT, Fano, Plasmonic, Transmission, refractive index

Posted Date: May 19th, 2021

DOl: https://doi.org/10.21203/rs.3.rs-461429/v1

License: (c) (1) This work is licensed under a Creative Commons Attribution 4.0 International License.

Read Full License

Version of Record: A version of this preprint was published at Plasmonics on December 3rd, 2021. See the published version at https://doi.org/10.1007/s11468-021-01570-y. 


\title{
Engineering the optical response of the novel plasmonic binary nanohole array
}

\author{
Mahdi Javidnasab, ${ }^{\text {a, b, * }}$, Saeid Khesali Azadi,, , Majid Ahmadpouri Legha, ${ }^{\text {, }}$ Hamid Naghshara a
}

\author{
a.Department of Condensed Matter Physics, University of Tabriz, Tabriz, Iran \\ b. Young Researchers and Elite Club, Tabriz Branch, Islamic Azad University, Tabriz, Iran \\ *Email: Mahdijavidnasab@gmail.com
}

\begin{abstract}
The phenomenon of extraordinary optical transmission (EOT) due to its advantages has been considered by researchers in various applications and in recent years many efforts have been made to engineer these structures to get the best possible response for desired applications. In this work, the optical properties of novel binary gold nano-hole arrays are investigated theoretically. We engineered the optical response of the system by adjusting the ratio of contribution of surface plasmon polariton (SPP) to localized surface plasmon resonance (LSPR) through the manipulation of the geometrical properties. The changes in the topology of this nano-hole array affected the intensity and the wavelength of transmission peaks. The sensitivity of the optical response to the refractive index was also investigated. The designed structure is a good candidate for use as a polarization-independent optical label-free sensor.
\end{abstract}

Keywords: FDTD, EOT, Fano, Plasmonic, Transmission, refractive index

\section{Introduction}

Since the discovery of Extraordinary optical transmission (EOT) by the group of Ebbesen in 1998[1], this phenomena has attracted the attention of many researchers due to its widespread applications in sensing[2-4], color filters[5, 6], perfect absorbers[7, 8], metamaterials[9], lenses[10], etc.

The enhanced transmission in a metal film with a subwavelength hole array and its unique geometrical and optical properties was registered by Ebbesen et al.[1] which it was not expected by Bethe model[11]. The origin of EOT has been explained in the framework of surface plasmon resonance phenomena.

At frequencies lower than the plasma frequency the electromagnetic field is stored in the collective electron oscillations, raising the possibility for confinement of the incident field to sub-wavelength 
volumes. One approach to accomplish this is using a thin metal film. The resulting boundary conditions quantize the plasma oscillations, which cause to create surface plasmon polaritons (SPP)[12]. If the dimensions of the thin metal film are further reduced to smaller than the incident wavelength, the retardant effects will be negligible and the electrons throughout the metal oscillate in phase. This phenomenon is known as localized surface plasmon resonance (LSPR) and is usually excited in curved metal objects such as metal nanoparticles and cavities of various topologies, providing the topology of additional momentum to couple directly to incident light[13]. LSPR frequency is dependent on the shape, size, and dielectric constant of metal objects. The LSPR allows the incident light to be highly concentrated and results in an enhancement in the local electromagnetic field. LSPRs and SPPs can co-exist at specific wavelengths such that the coupling between them can contribute to the optimization of the nanohole arrays to get the desired optical response for different applications[14].

Fano resonance is a result of the interference of two different resonances. Thus, Fano-like nature of EOT peaks can be interpreted as a combination of a localized surface plasmon resonance inside single holes and propagating surface plasmon resonance on the surface of the plasmonic thin film $[15,16]$.

The Fano nature of resonances which leads to asymmetric line shape of the EOT peaks has been proved[17]. Fano resonance has been of great interest to researchers for many applications such as color filters[6, 18], photonic crystals[19, 20], metamaterials[21, 22], surface enhanced Raman spectroscopy (SERS)[17, 23, 24] and sensors[17, 18, 25, 26] due to its very narrow asymmetric signal shape and its effect on the strong enhancement of the electromagnetic fields.

Therefore, by fitting Fano peaks and finding the Fano parameter of them more detailed information about the nature of different optical responses for various nanostructure configurations can be achieved.

In addition to works done to investigate the physical roots of this phenomenon, there are also several works[11, 27-29] in which attempts were made to manipulate the topology of these systems to achieve the controlled optical responses for different applications.

In this study, we designed and numerically simulated a novel binary nanohole array for extraordinary optical transmission applications. The structure consists of a gold thin film with binary nanohole array on a glass substrate. We studied the effect of different geometrical parameters on the transmittance spectral characteristics through this binary nanohole array. Besides, we discussed Fano properties of the main EOT peak as a next step for understanding the physical bases of geometrical parameters changes nanostructure designed by us on optical response more deeply.

\section{Simulation method}


In this paper, the numerical analysis of light interaction with the proposed nanohole array has been carried out using the 3D finite difference time domain (FDTD) approach.

FDTD is one of the most used methods to solve Maxwell's time-dependent equations numerically, particularly when obtaining an analytical solution is extremely hard or even impossible[18].

Normal incidence zero-order transmission spectra were simulated. In our geometry, the light first passes through the array of metal holes and then glass.

The refractive indexes were set equal to 1.0 for air and 1.49 for substrate (glass from albite or PMMA). The periodic boundary conditions have been applied in $\mathrm{x}$ and $\mathrm{y}$-directions in a unit cell of the nanohole array. This lets us obtain the optical response of the entire system in a much shorter time scale. In the z-direction of the incident plane wave propagation, perfectly matched layers (PML) were utilized.

\section{Results and discussion}

Here, we investigate the evolution of plasmonic properties of binary nanohole array using transmittance spectra. The metal nanostructure consists of two holes in different sizes with large and smaller diameters ( $D 1$ and $D 2$, respectively) in the unit cell which is schematically shown in figure 1 .

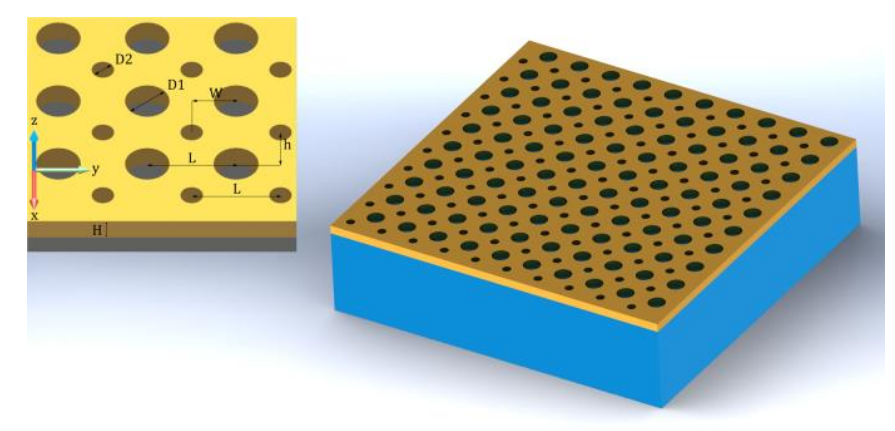

Figure 1. Schematics diagram and morphology geometry of binary nano-hole array structure.

The diameter of the larger hole is fixed. The diameter of the smaller holes is variated and calculated as $D 2=\alpha \times \mathrm{D} 1$ where $\alpha$ is a real number between 0 and 1 . The periodic length of the hole array is shown with $L$ and equals to the minimum distance of the origin of two same neighbors (either large or small) holes. The simplest unit cell is denoted in Figure 1 with the unit cell parameter $W$ is the distance between large and small holes, measured along the $\mathrm{x}$-axis. Constant parameters are 
defined as large hole diameter $D 1=100 \mathrm{~nm}, L=400 \mathrm{~nm}$, thickness $H=200 \mathrm{~nm}$, and $h=$ $200 \mathrm{~nm}$ (see Figure 1). The gold film thickness is chosen optimal and equal to $200 \mathrm{~nm}$ based on previous works[30-32].

The effects of other structural parameters on the optical response of the binary nanohole array are presented in the separated sections. In the first two sections, the effect of the $\alpha$ and $W$ on the transmission spectrum of a p-polarized light has been discussed. In the next, the effect of asymmetry on the transmission spectra of different polarizations state of illuminated light has been analyzed. And Finally, as an example of applications of such a system, we studied its sensing properties by varying different geometrical parameters.

\section{I: effect of hole size on optical transmission}

The optical transmission spectrum of the binary nanohole array for different values of $\alpha$ is shown in Figure 2.a. For $\alpha=0$ the most amplitude asymmetric peak is located at $446 \mathrm{THz}$, also there is a wide peak, which has a lower amplitude. As $\alpha$ increases, the width of the EOT peak decreases, which means better resolution in the case of using nano hole array as a refractive index sensitive sensor.

(a)

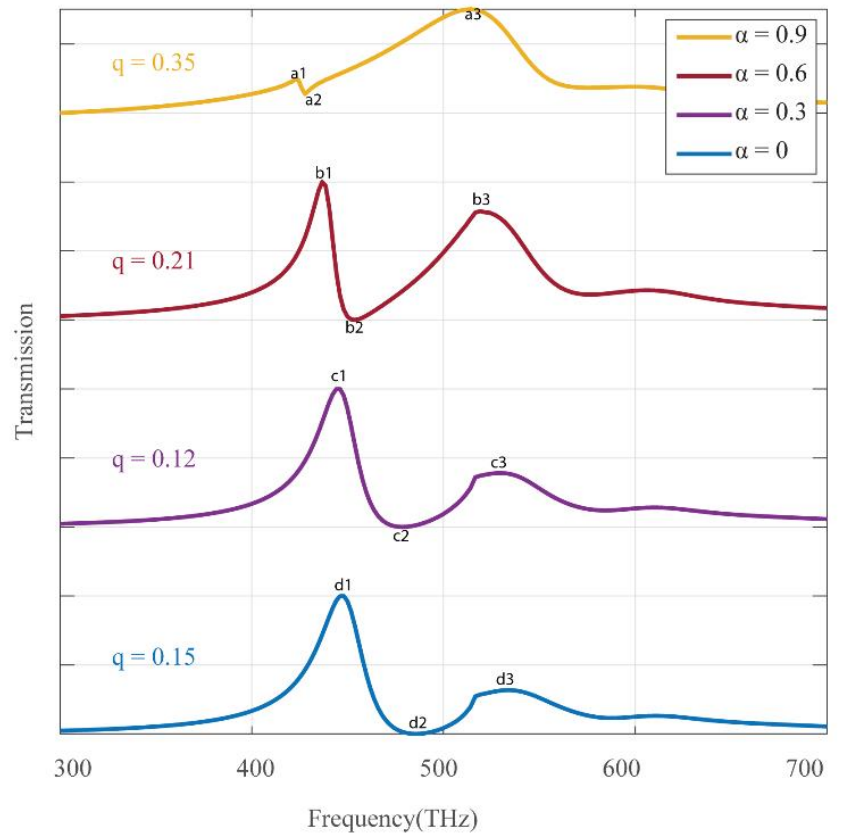

(b)

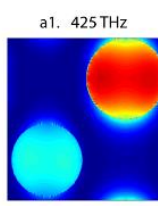

b1. $437 \mathrm{THz}$

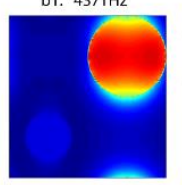

c1. $444 \mathrm{THz}$

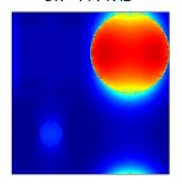

d1. $446 \mathrm{THz}$

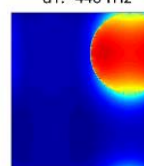

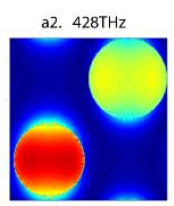

b2. $454 \mathrm{THz}$

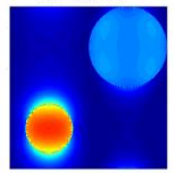

c2. $479 \mathrm{THz}$

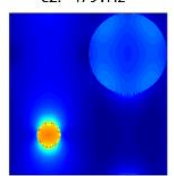

d2. $486 \mathrm{THz}$

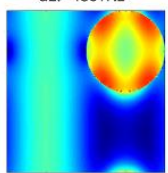

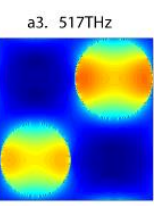

b3. $519 \mathrm{THz}$

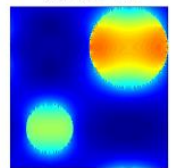

c3. $530 \mathrm{THz}$

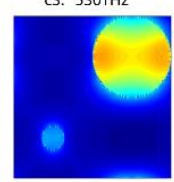

d3. $535 \mathrm{THz}$

Figure 2. (a) Transmission spectra of binary nanohole array structure (b) electric field distribution in the middle of gold layer for peaks and dips, at four different configurations with different $\alpha$ values including $\alpha=0,0,3,0,6$ and 0,9 while $W$ is fixed and equals to $200 \mathrm{~nm}$ for all the configurations. 
The formation of multipolarity in the dip frequency is visible to all cases in figure 2.b., which confirms the hypothesis that LSPR leads to scattering or absorption resulting in transmission dips[33]. These kinds of dips imply an asymmetry in the shape of EOT peaks. The Asymmetry in the shape of the curve is a characteristic of the Fano peaks. According to Figure 2.a, with an increase in $\alpha$, the amplitude of the Fano peak is decreased, whereas for $\alpha=1$ it has been disappeared. Also, the plot shows that the Fano transmission peak shifts toward blue as $\alpha$ decreases.

The asymmetric line shape of a Fano peak can be described by the well-known Fano formula:

$$
F(\omega)=A+F \frac{\left[q+2\left(\omega-\omega_{0}\right) / \Gamma\right]^{2}}{1+\left[2\left(\omega-\omega_{0}\right) / \Gamma\right]^{2}}
$$

Where $A$ and $F$ are constant factors, $\omega_{0}$ and $\Gamma$ are resonance frequency and resonance linewidth respectively, and $q$ is the Fano parameter which accounts for lineshape asymmetry.

We fitted Fano peaks of simulated transmission spectra with the mentioned Fano formula to obtain $q$ parameter for them. Comparison of simulated spectra with analytical equation (2) is presented in Figure 3 for $\alpha=0.3$ and $W=200 \mathrm{~nm}$. If we ignore $\alpha=0$, for other values of $\alpha$, as $\alpha$ increases $q$ gets larger which means asymmetry of the resonance lineshape is increased.

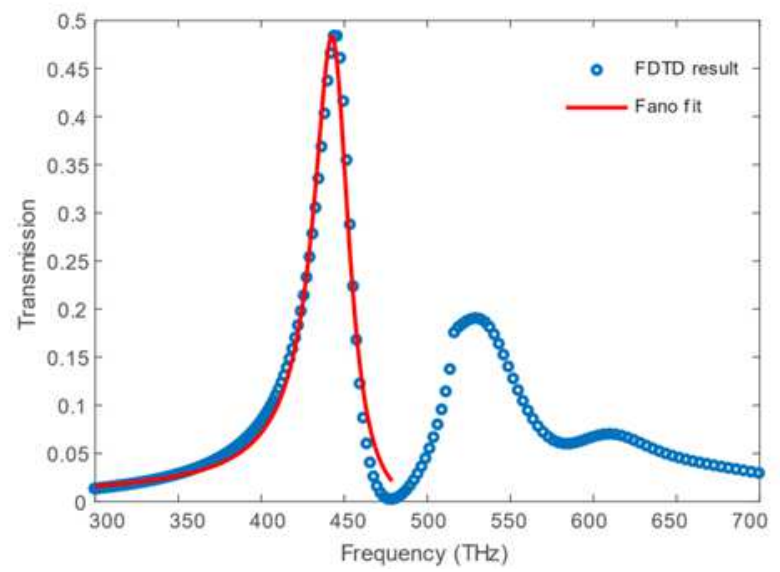

Figure 3. Fano model fit (solid curve) to the FDTD transmission data (Circles) for the of binary nanohole array in a gold film.

\section{II: Effect of $W$}


In this section, we investigated the influence of changing the distance between the holes along the $y$-axis ( $W$ parameter) and fixed $\alpha$ parameter with a value equal to 0.7 on transmission spectra of our binary nanohole array structure. The presented binary nanohole array structure is periodical, therefore, the value range for parameter $W$ is changed from 0 to just half of the structure period $\mathrm{L}$.

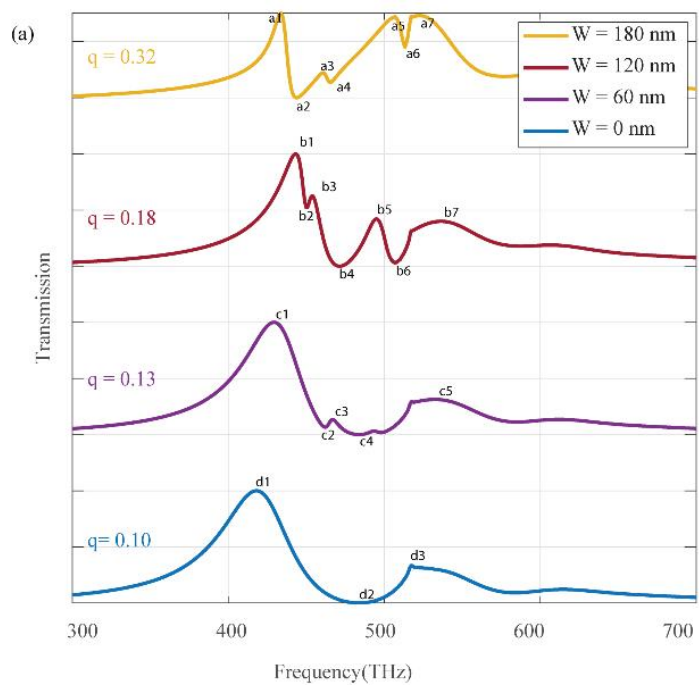

(b)

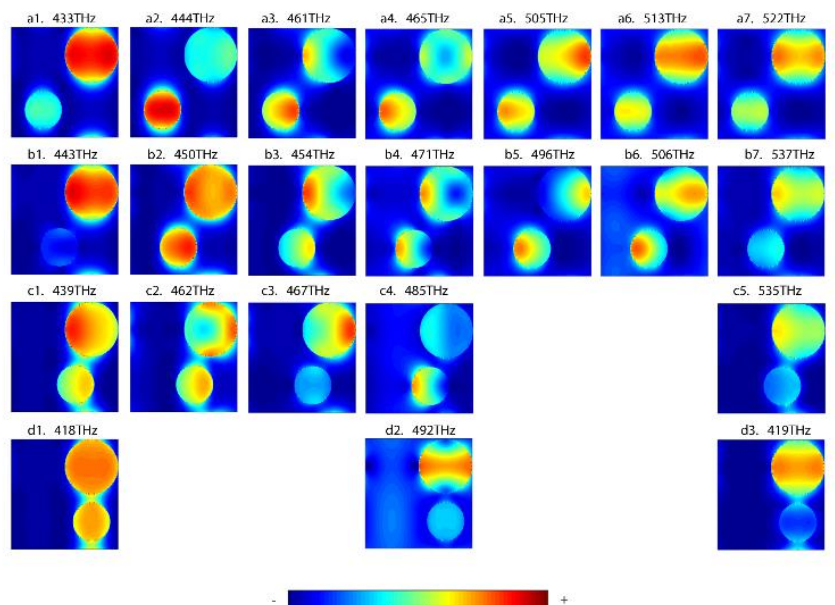

Figure 4. (a) Transmission spectra of binary nanohole array structure (b) electric field distribution in the middle of gold layer for peaks and dips, at four different configurations with different $W$ values including $W=0,0,60,120$ and 180 while $\alpha$ is fixed and equals to 0.7 for all the configurations.

Transformation in transmission spectral characteristics for different values of parameter $W$ is shown in Figure 2.b. The EOT peak with a spectral position around $418 \mathrm{THz}$ has its maximum intensity for $W=0$. With this $W$ parameter value for nanostructure from Figure 1 its morphology transformed into two holes located in a single column along the x-axis. For $W=120 \mathrm{~nm}$, the main Fano transmission peak shifted to high frequencies (blue shift) with decreasing its amplitude. As a result, the new peaks with low intensity at 454, 496, and $537 \mathrm{THz}$ are observed. Further increasing of $W$ leads to the decreasing of main Fano peak amplitude and increasing of other Fanolike peaks amplitude. Changing $W$ from 0 to $L / 2$ leads to an increase of the amplitude of the main Fano peak almost two times.

Figure 2.b. shows electric field distribution in the middle of gold layer for peaks and dips, at four different configurations with different $W$ values including $W=0,0,60,120$ and 180 while $\alpha$ is fixed and equals to 0.7 for all the configurations. For $W=0$ and 0,60 the electric fields inside two holes are coupled so that we can define them as a single hole with an effective radius, that is why there is only one reliable peak in the transmission spectrum. For $W=120$ and 180, the electric field inside two different holes are independent which leads to the formation of different resonance modes, resulting in more peaks that can be useful in the surface enhanced Raman spectroscopy (SERS) applications[34]. 


\section{III: Polarization dependence}

The use of polarization of light is an emerging research field that has progressed significantly in recent years within the biomedical field[35-38]. In this section, we are investigating the response of our proposed design to the changes in the polarization of incident light.

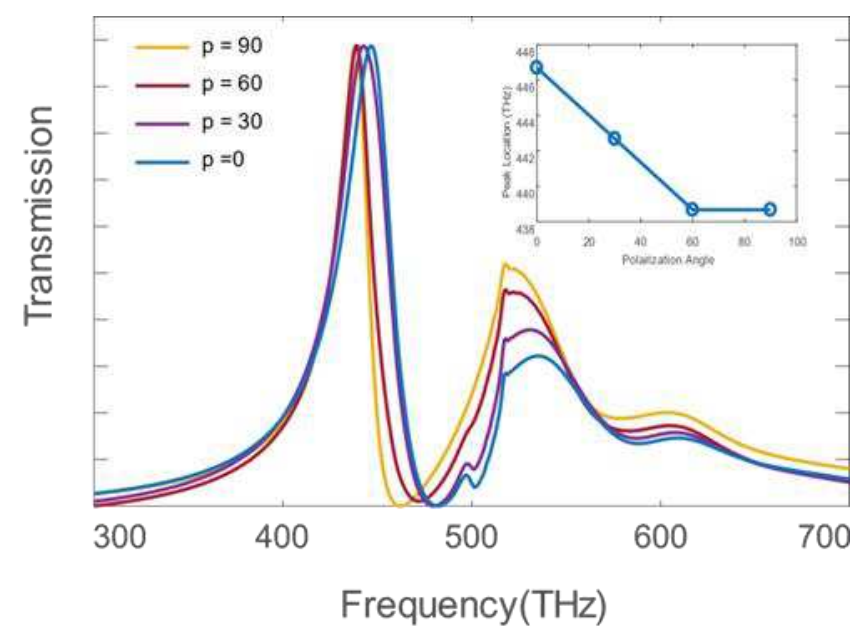

Figure 5. Transmission spectra of Au binary nano hole array for different polarization angels and $\alpha=0.5$ and $W=100 \mathrm{~nm}$.

Here we have found that the position of spectral peaks is almost nonsensitive for different polarization states when symmetrical nanostructure with $W=L / 2$ is considered. The other values of the $W$ parameter led to the asymmetry of nanostructure and the influence of different polarization states on the transmission spectrum is observed. The polarization features of transmission spectra for binary nanohole array structure with structural parameter $W=100 \mathrm{~nm}$ and different polarization states of incident light were shown in Figure 5.

For different polarization states with the component of electrical field oscillated along the x-axis for incidence light the transmission spectra with main Fano-like peak around $520 \mathrm{THz}$ are obtained. For p-polarized light, the p parameter equals to $0^{0}$ and for s-polarized $90^{\circ}$. However, changing the polarization angle from 0 to larger angles leads to an increase in the y-component of the electrical field and a decrease in the x-component of the electrical field, while the amplitude of Fano peak increases. Thus, the changes in the symmetry of the nanohole array structure by shifting the position of the holes, influence on FWHM parameter of Fano peaks for transmission spectra.

The FWHM of Fano peak reduces due to the continuous increase of the polarization angle from zero to 90 degrees. The shifts in transmission spectra caused by different polarization angles of incident light are negligible for sensory application. 


\section{IV: Refractive index sensitivity}

One of the most important applications of the nanohole arrays is sensing. The performance of a sensor can be evaluated by the figure of merit (FOM) which is the ratio of refractive index sensitivity to $F W H M$. For four different morphology of binary nanohole arrays structure, the sensitivity was simulated. Figure 6 shows the sensitivity and shift of EOT peaks for four morphology of nanostructure for detection of different medium with refractive index $n=1-1.5$ $R I U$. The transmission spectra for the combination of morphology parameters $\alpha=0$ and $\alpha=0.7$ and $W=0$ are shown in Figure 6.a and 6.b were determined average FOM values equal to 0.8053 and 0.3561 , respectively. Increasing of $W$ parameter influences the gradual decrease of the main transmission peak intensity. This result is a perspective for sensors based on amplitude measuring. The new Fano-like peaks for $\alpha=0.7$ and $W=100 \mathrm{~nm}$ with a spectral position near $490 \mathrm{THz}$ and average FOM equal to 0.5338 were determined (Figure 6.c). For $\alpha=0.7$ and $W=200 \mathrm{~nm}$, the amplitude of the transmission peaks has reached its lowest value in comparison with three previous combinations of morphology parameters.

The largest average FOM among all structural configurations equal to 0.8185 due to the regular shape of EOT peak is obtained (Figure 6.d).

The high refractive-index sensitivity of these nanostructures makes them suitable for use in chemical and biological sensing for gaseous and liquid mediums. 
(a)

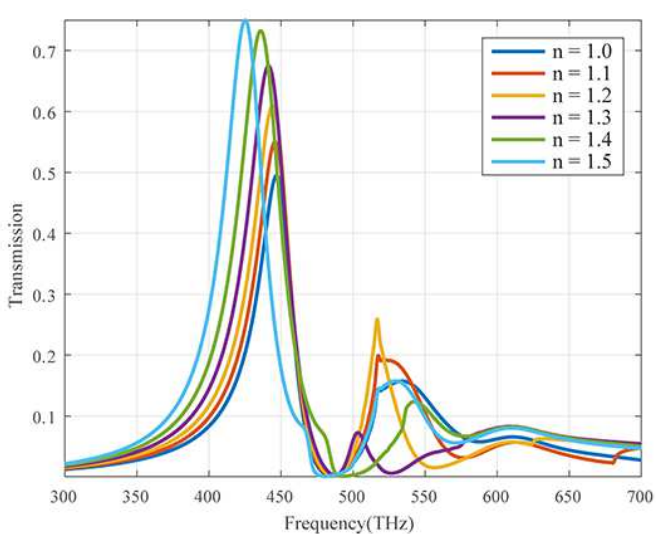

(c)

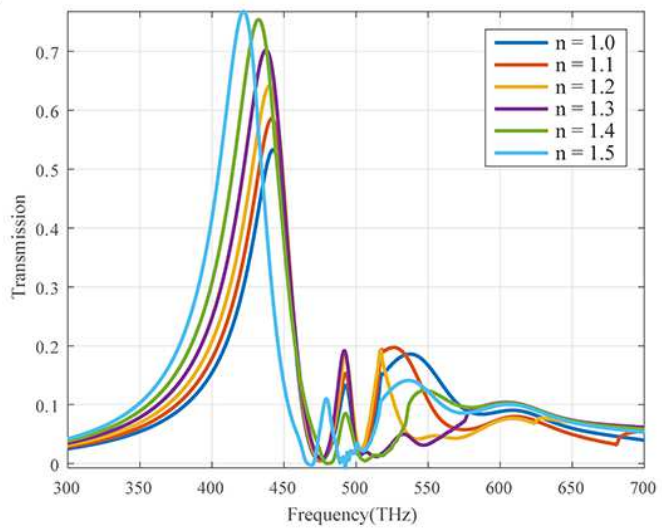

(b)

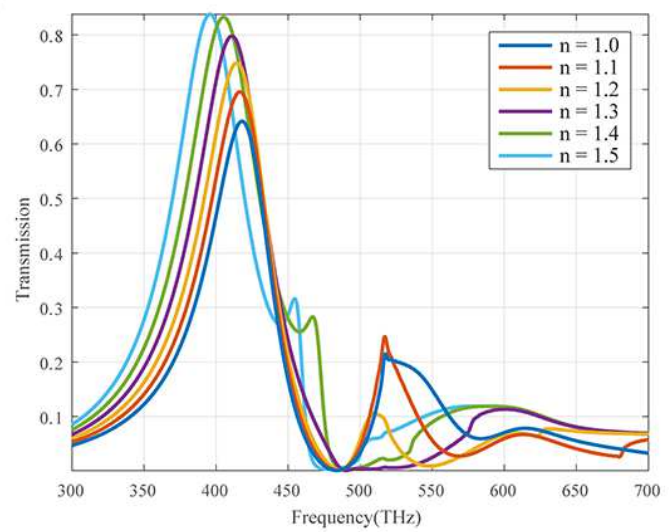

(d)

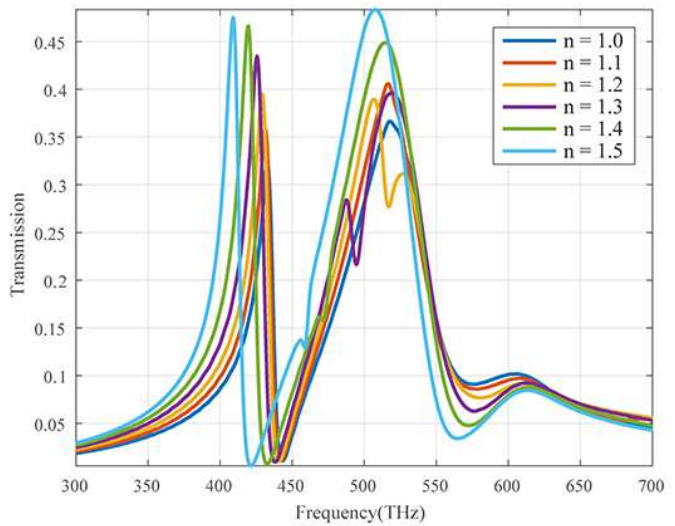

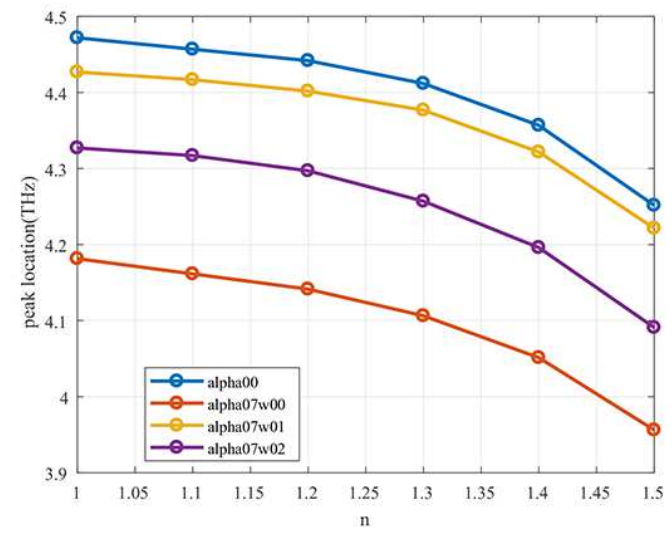

Figure 6. Refractive index sensitivity (a) $\alpha=0$ (b) $\alpha=0.7$ and $W=0$ (c) $\alpha=0.7$ and $W=100 \mathrm{~nm}$ (d) $\alpha=0.7$ and $W=200 \mathrm{~nm}$. (e) Refractive index dependency of spectral peak position.

\section{Conclusions}

Novel binary nanohole array structure with tunable optical parameters was developed and analyzed in detail. The main peak in transmission spectra is identified with Fano-like nature resonance. The tunability of transmission spectra is carried out by the variation of nanoholes surface density. These spectral transformations occur under changing the ratio of the contribution of localized surface 
plasmon resonance excited in single holes and non-localized surface plasmon resonance excited on the up/down flat surfaces. The increasing of structural parameters, such as the $\alpha$ and $W$ leads to Fano parameter increases in range values from $q=0.12$ to 0.35 for different $\alpha$, as well as from $q=0.10$ to 0.32 for different values of $W$ (for the case when $\alpha=0.7$ ) was shown. Thus, simulation results demonstrate the blue wavelength shift in the Fano-like transmission spectra associated with the increasing of $\alpha$ or $W$ parameters.

The application possibility of developed the structure for optical sensors has been shown for various configurations of the morphology. The sensitivity for changing of detection medium is calculated. The unique morphology configuration combines with properties of Fano-like resonance of nanohole arrays are perspective for plasmonic optical sensors and nanoelectromechanical systems.

*Funding: no funding received for this work.

*Conflicts of interest/Competing interests: there are no conflicts to declare.

*Availability of data and material: The data created by the simulations during the current study are available from the corresponding author on reasonable request.

* Code availability: The simulation codes wrote for the current study are available from the corresponding author on reasonable request.

*Authors' contributions: Mahdi Javidnasab provided the idea of designing binary shaped nanohole array. Mahdi Javidnasab, Saeid Khesali Azadi and Majid Ahmadpouri Legha did the calculations and data reperesentations. Dr. Hamid naghshara helped to explain the physics behind the optical response of proposed structure.

*Ethics approval: not applicable.

*Consent to participate: not applicable.

*Consent for publication: not applicable.

\section{References}

1. Ebbesen TW, Lezec HJ, Ghaemi HF, et al (1998) Extraordinary optical transmission through sub-wavelength hole arrays. Nature 391:667-669. https://doi.org/10.1038/35570

2. Escobedo C (2013) On-chip nanohole array based sensing: A review. Lab Chip

3. O'Hara JF, Withayachumnankul W, Al-Naib I (2012) A review on thin-film sensing with terahertz waves. J. Infrared, Millimeter, Terahertz Waves

4. Lee Y, Song H, Ahn H, et al (2018) Rapid and real-time diagnosis of hypoalbuminemia using an extraordinary optical transmission biosensor. Sensors Actuators, B Chem. https://doi.org/10.1016/j.snb.2018.07.119

5. Inoue D, Miura A, Nomura T, et al (2011) Polarization independent visible color filter comprising an aluminum film with surface-plasmon enhanced transmission through a subwavelength array of holes. Appl Phys Lett. https://doi.org/10.1063/1.3560467

6. Wang LS, Zeng B, Hu XL, et al (2015) Effect of relative nanohole position on colour purity of ultrathin plasmonic subtractive colour filters. Nanotechnology 26:305204. 
https://doi.org/10.1088/0957-4484/26/30/305204

7. Braun J, Gompf B, Kobiela G, Dressel M (2009) How holes can obscure the view:

Suppressed transmission through an ultrathin metal film by a subwavelength hole array. Phys Rev Lett. https://doi.org/10.1103/PhysRevLett.103.203901

8. Yu F, Wang H, Zou S (2010) Efficient and tunable light trapping thin films. J Phys Chem C. https://doi.org/10.1021/jp909974h

9. Chen WC, Landy NI, Kempa K, Padilla WJ (2013) Optical Transmission: A Subwavelength Extraordinary-Optical-Transmission Channel in Babinet Metamaterials (Advanced Optical Materials 3/2013). Adv Opt Mater.

https://doi.org/10.1002/adom.201370017

10. Fan F, Chen S, Wang X-H, Chang S-J (2013) Tunable nonreciprocal terahertz transmission and enhancement based on metal/magneto-optic plasmonic lens. Opt Express. https://doi.org/10.1364/oe.21.008614

11. Wang W, Lu Y, Knize RJ, et al (2009) Tunable and polarization-selective THz range transmission properties of metallic rectangular array with a varying hole channel shape. Opt Express. https://doi.org/10.1364/oe.17.007361

12. Maier SA (2007) Plasmonics: Fundamentals and applications

13. Zayats A V., Smolyaninov II, Maradudin AA (2005) Nano-optics of surface plasmon polaritons. Phys. Rep. 408:131-314

14. Du L, Zhang X, Mei T, Yuan X (2010) Localized surface plasmons, surface plasmon polaritons, and their coupling in 2D metallic array for SERS. Opt Express 18:1959-1965. https://doi.org/10.1364/oe.18.001959

15. Fano U (1961) Effects of configuration interaction on intensities and phase shifts. Phys Rev. https://doi.org/10.1103/PhysRev.124.1866

16. Kamenetskii E, Sadreev A, Miroshnichenko A (2018) Fano Resonances in Optics and Microwaves. Springer International Publishing, Cham

17. Altewischer E, van Exter MP, Woerdman JP (2005) Analytic model of optical depolarization in square and hexagonal nanohole arrays. J Opt Soc Am B 22:1731. https://doi.org/10.1364/JOSAB.22.001731

18. Zhao E, Jia P, Ebendorff-Heidepriem H, et al (2017) Localized surface plasmon resonance sensing structure based on gold nanohole array on beveled fiber edge. Nanotechnology 28:435504. https://doi.org/10.1088/1361-6528/aa847a

19. Yu Y, Xue W, Semenova E, et al (2017) Demonstration of a self-pulsing photonic crystal Fano laser. Nat Photonics. https://doi.org/10.1038/nphoton.2016.248

20. Wang W, Klots A, Yang Y, et al (2015) Enhanced absorption in two-dimensional materials via Fano-resonant photonic crystals. Appl Phys Lett. https://doi.org/10.1063/1.4919760

21. Xiao S, Wang T, Jiang X, et al (2017) Strong interaction between graphene layer and Fano resonance in terahertz metamaterials. J Phys D Appl Phys. https://doi.org/10.1088/13616463/aa69b1

22. Yang Y, Wang W, Boulesbaa A, et al (2015) Nonlinear Fano-Resonant Dielectric Metasurfaces. Nano Lett. https://doi.org/10.1021/acs.nanolett.5b02802

23. King NS, Liu L, Yang X, et al (2015) Fano Resonant Aluminum Nanoclusters for Plasmonic Colorimetric Sensing. ACS Nano. https://doi.org/10.1021/acsnano.5b04864

24. Huang Y, Zhang X, Ringe E, et al (2018) Detailed correlations between SERS enhancement and plasmon resonances in subwavelength closely spaced Au nanorod 
arrays. Nanoscale 10:4267-4275. https://doi.org/10.1039/C7NR08959G

25. Zheng G, Zou X, Chen Y, et al (2017) Fano resonance in graphene-MoS2 heterostructurebased surface plasmon resonance biosensor and its potential applications. Opt Mater (Amst). https://doi.org/10.1016/j.optmat.2017.02.001

26. Lee K-L, Huang J-B, Chang J-W, et al (2015) Ultrasensitive Biosensors Using Enhanced Fano Resonances in Capped Gold Nanoslit Arrays. Sci Rep 5:8547.

https://doi.org/10.1038/srep08547

27. Balaur E, Sadatnajafi C, Kou SS, et al (2016) Continuously Tunable, Polarization Controlled, Colour Palette Produced from Nanoscale Plasmonic Pixels. Sci Rep. https://doi.org/10.1038/srep28062

28. Yue W, Wang Z, Yang Y, et al (2014) Enhanced extraordinary optical transmission (EOT) through arrays of bridged nanohole pairs and their sensing applications. Nanoscale. https://doi.org/10.1039/c4nr01001a

29. Anh ND, Chun BJ, Choi S, et al (2019) Plasmonic dynamics measured with frequencycomb-referenced phase spectroscopy. Nat. Phys.

30. Genet C, Van Exter MP, Woerdman JP (2003) Fano-type interpretation of red shifts and red tails in hole array transmission spectra. Opt Commun 225:. https://doi.org/10.1016/j.optcom.2003.07.037

31. Kang ESH, Ekinge H, Jonsson MP (2019) Plasmonic fanoholes: on the gradual transition from suppressed to enhanced optical transmission through nanohole arrays in metal films of increasing film thickness. Opt Mater Express 9:. https://doi.org/10.1364/ome.9.001404

32. Mahros AM, Tharwat MM (2015) Investigating the fabrication imperfections of plasmonic nanohole arrays and its effect on the optical transmission spectra. J Nanomater 2015:. https://doi.org/10.1155/2015/178583

33. Malani S B, Viswanath P (2018) Impact of ordering of gold nanohole arrays on refractive index sensing. J Opt Soc Am B 35:. https://doi.org/10.1364/josab.35.002501

34. Kasani S, Zheng P, Wu N (2018) Tailoring Optical Properties of a Large-Area Plasmonic Gold Nanoring Array Pattern. J Phys Chem C 122:

https://doi.org/10.1021/acs.jpcc.7b11660

35. Haider F, Mashrafi M, Haider R, et al (2020) Asymmetric core-guided polarizationdependent plasmonic biosensor. Appl Opt 59:. https://doi.org/10.1364/ao.400301

36. Wang J, Pei L, Wu L, et al (2020) A Polarization-independent SPR Sensor Based on Photonic Crystal Fiber for Low RI Detection. Plasmonics 15:.

https://doi.org/10.1007/s11468-019-01054-0

37. Yesilkoy F, Terborg RA, Pello J, et al (2018) Phase-sensitive plasmonic biosensor using a portable and large field-of-view interferometric microarray imager. Light Sci Appl 7:. https://doi.org/10.1038/lsa.2017.152

38. Sharma Y, Gupta S, Das A, Dhawan A (2020) Polarization-independent SERS substrates for trace detection of chemical and biological molecules 
Figures
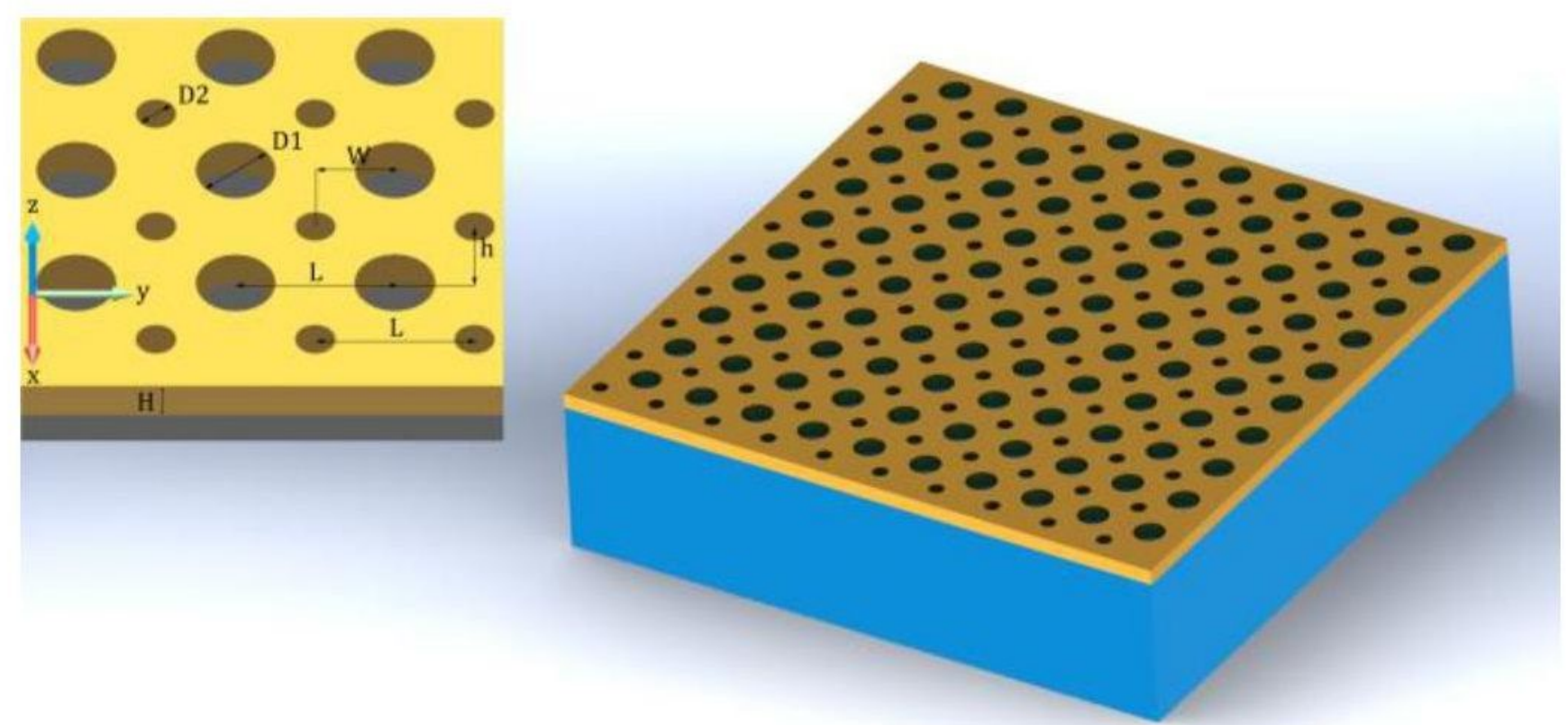

Figure 1

Schematics diagram and morphology geometry of binary nano-hole array structure 
(a)

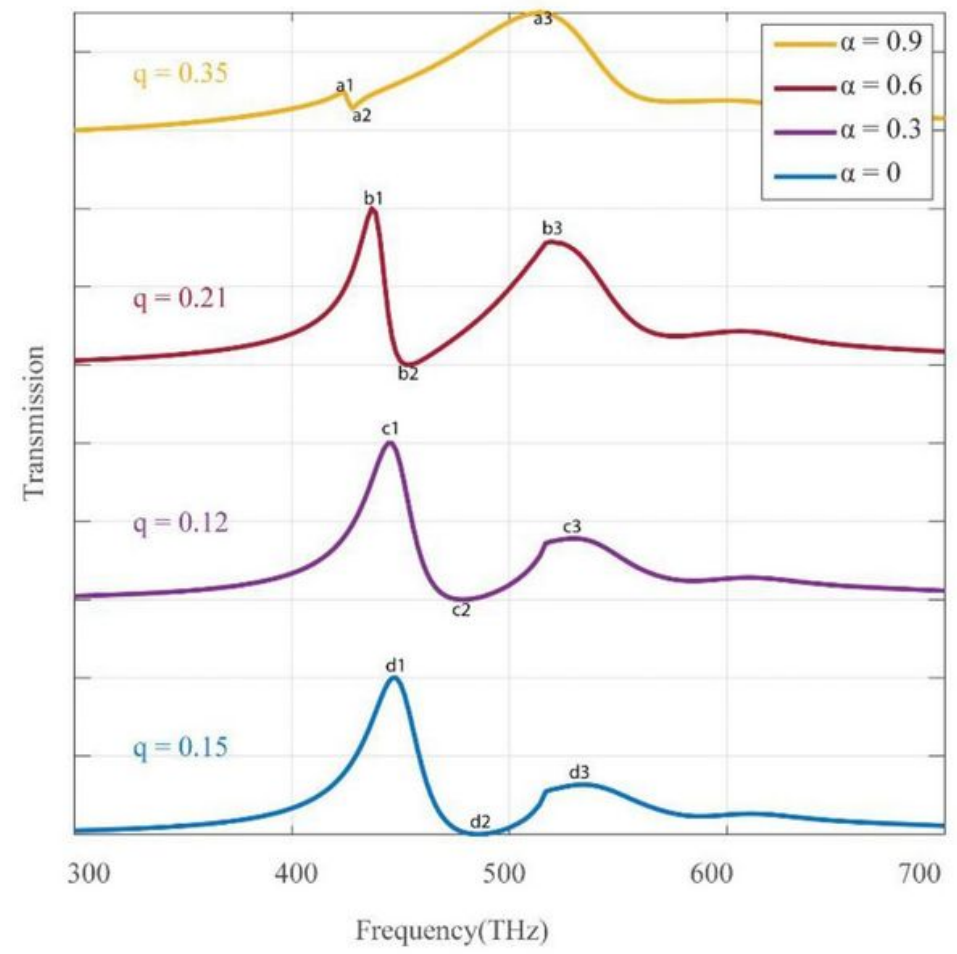

a1. $425 \mathrm{THz}$

(b)

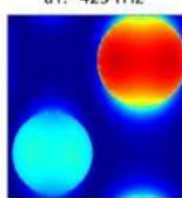

b1. $437 \mathrm{THz}$

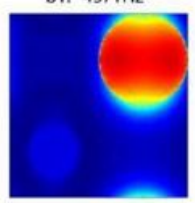

c1. $444 \mathrm{THz}$

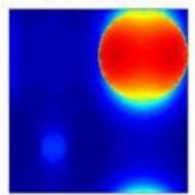

d1. $446 \mathrm{THz}$

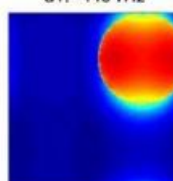

a2. $428 \mathrm{THz}$

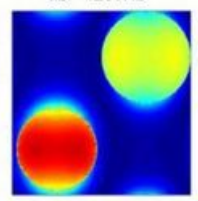

b2. $454 \mathrm{THz}$

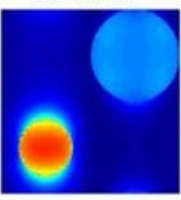

C2. $479 \mathrm{THz}$

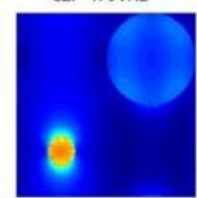

d2. $486 \mathrm{THz}$

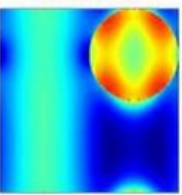

a3. $517 \mathrm{THz}$

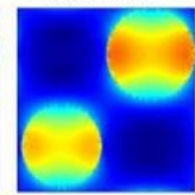

b3. $519 \mathrm{THz}$

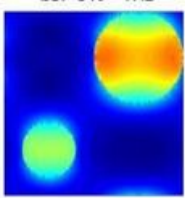

c3. $530 \mathrm{THz}$

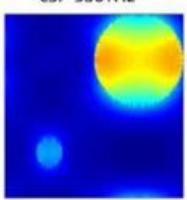

d3. $535 \mathrm{THz}$

\section{Figure 2}

(a) Transmission spectra of binary nanohole array structure (b) electric field distribution in the middle of gold layer for peaks and dips, at four different configurations with different $\nabla$ values including $\nabla=0,0.3$,

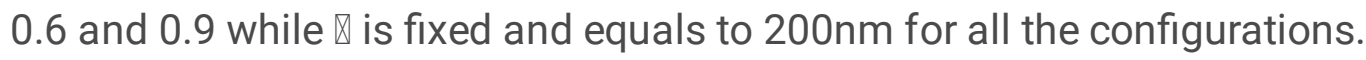




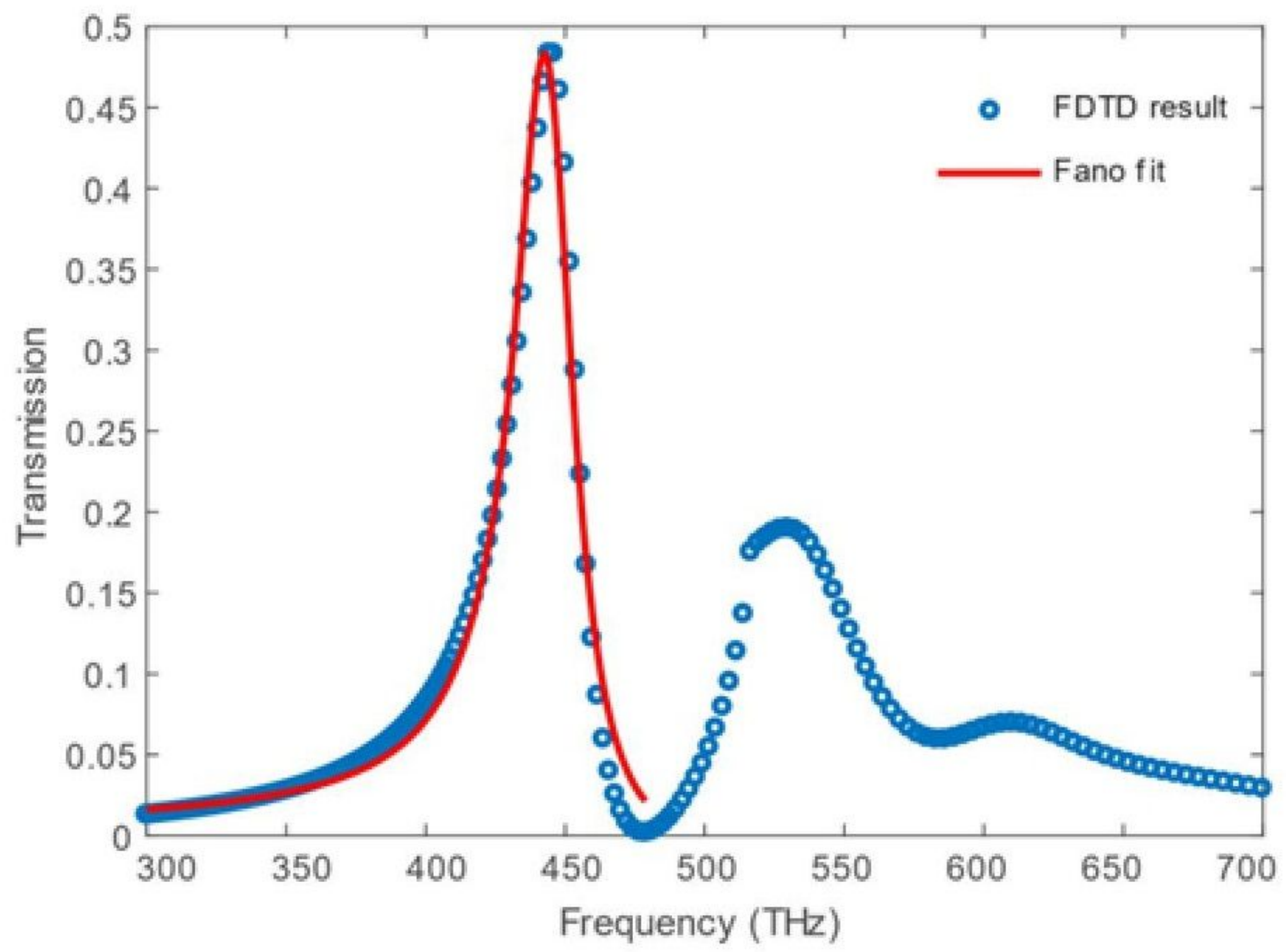

Figure 3

Fano model fit (solid curve) to the FDTD transmission data (Circles) for the of binary nanohole array in a gold film.

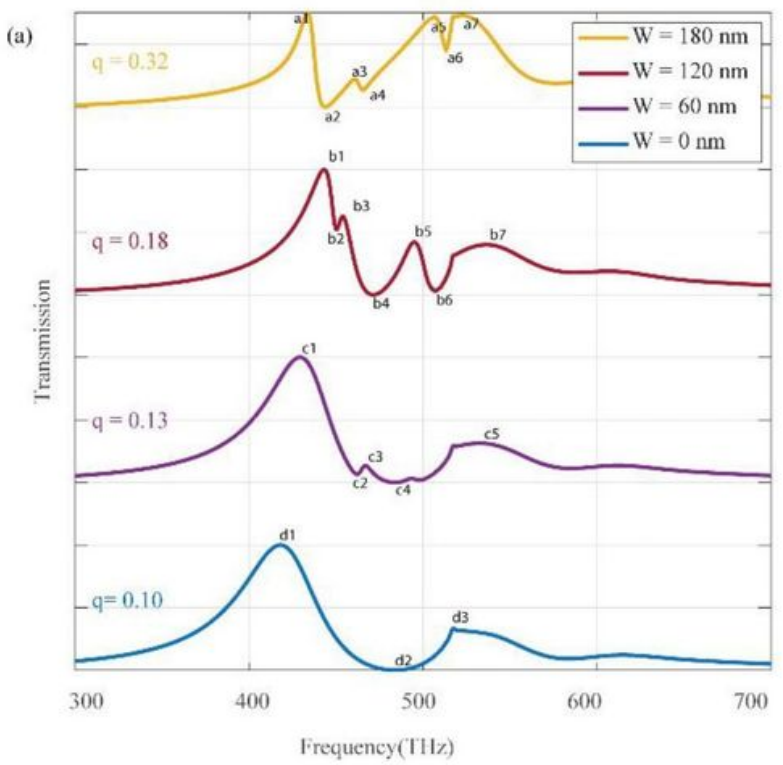

(b)

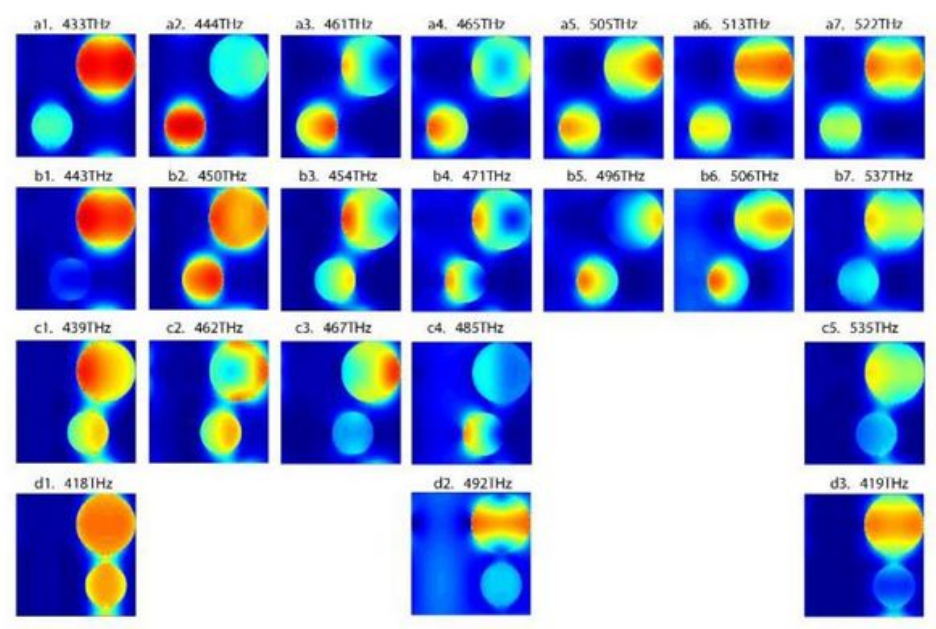


Figure 4

(a) Transmission spectra of binary nanohole array structure (b) electric field distribution in the middle of gold layer for peaks and dips, at four different configurations with different $\nabla$ values including $\nabla=0,0.60$, 120 and 180 while $\otimes$ is fixed and equals to 0.7 for all the configurations.

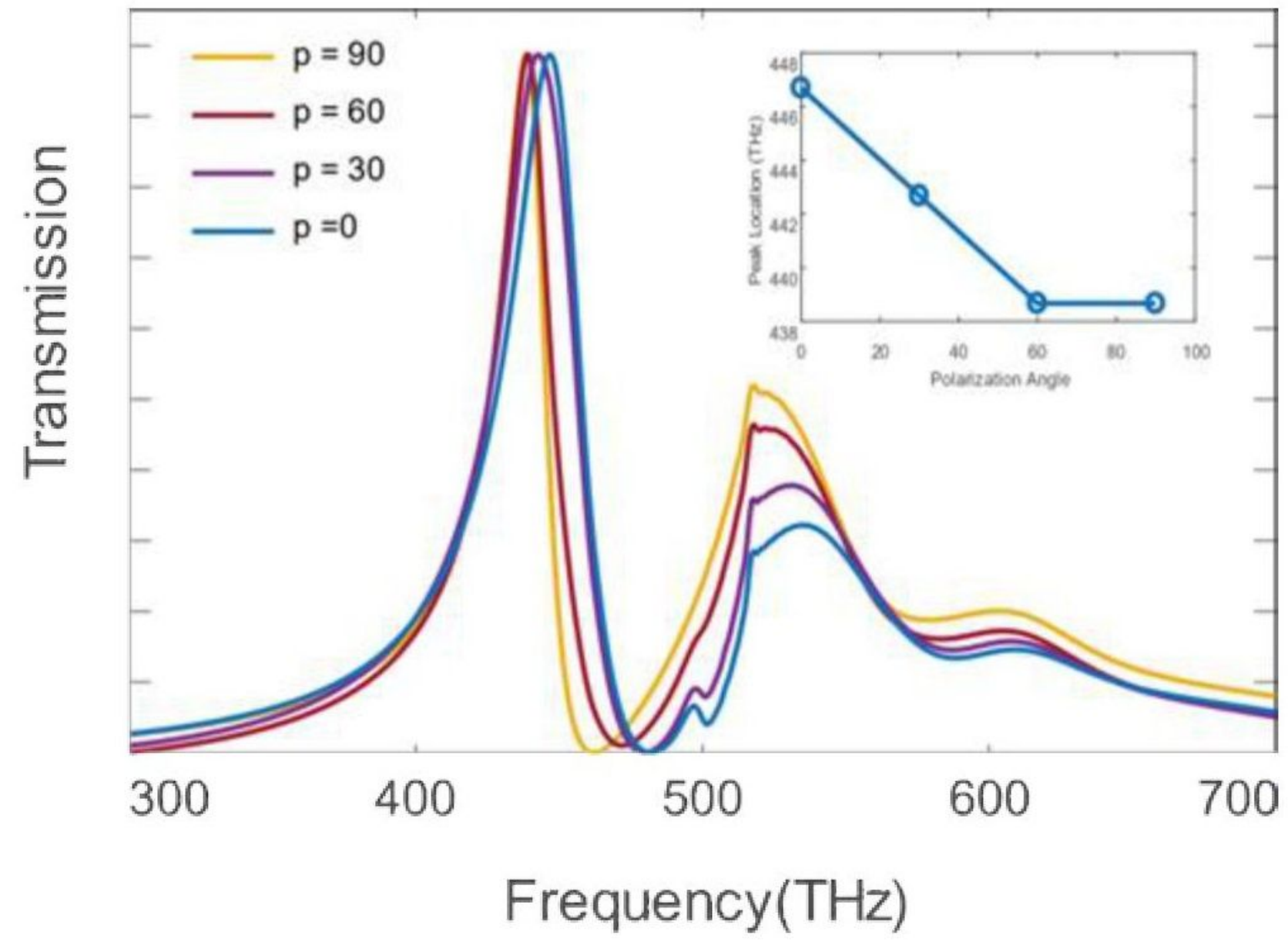

Figure 5

Transmission spectra of Au binary nano hole array for different polarization angels and $\nabla=0.5$ and $\nabla=$ 100 怄. 
(a)

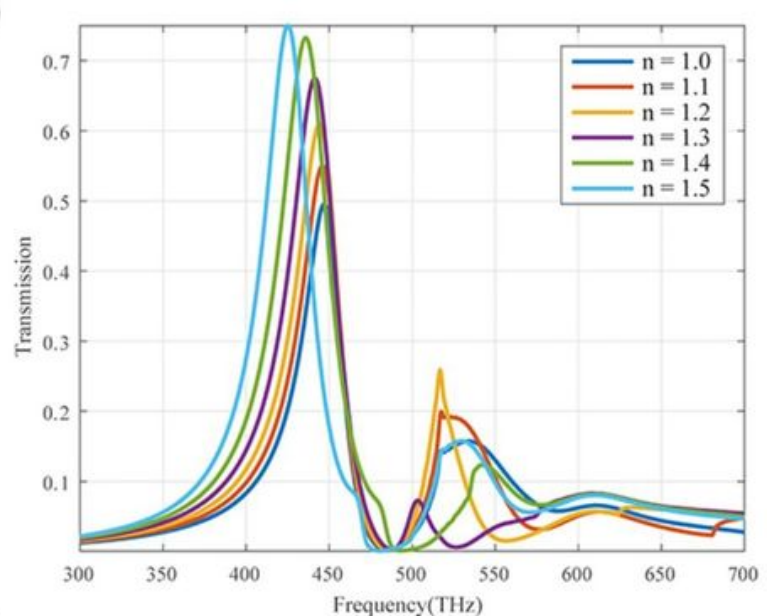

(c)

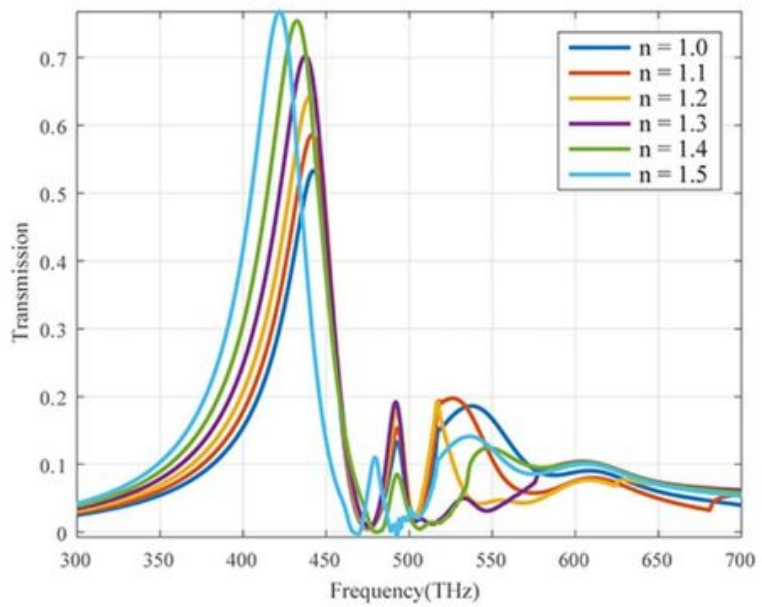

(b)

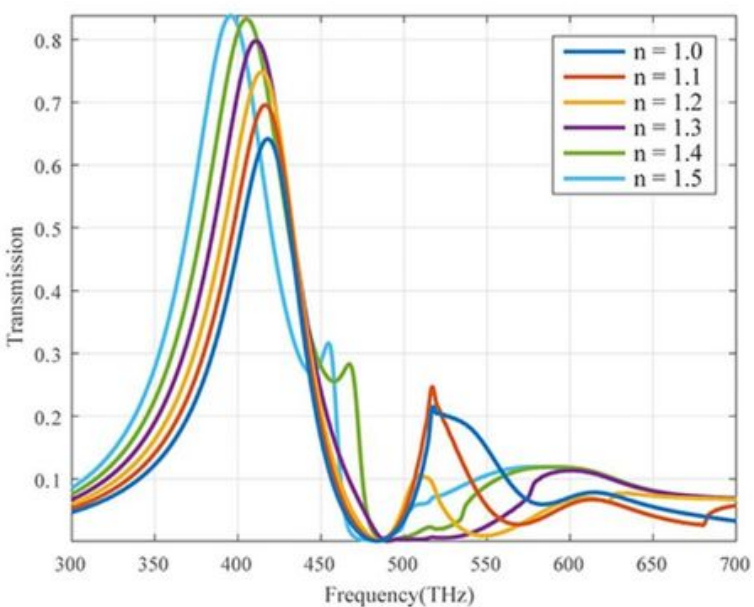

(d)

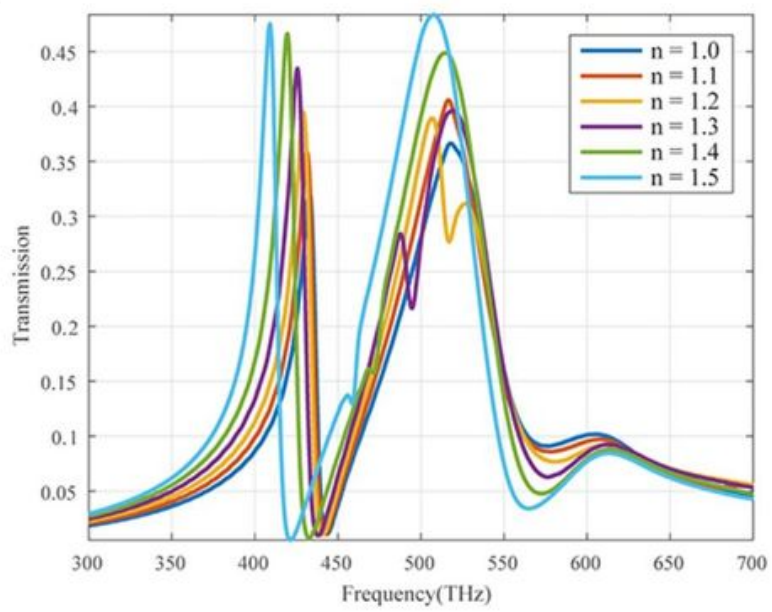

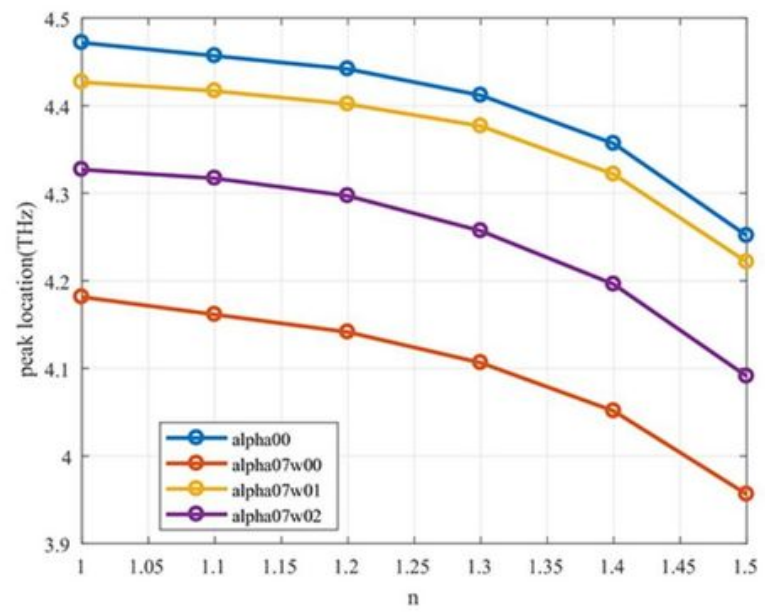

Figure 6

Refractive index sensitivity (a) $\nabla=0$ (b) $\nabla=0.7$ and $\nabla=0$ (c) $\nabla=0.7$ and $\nabla=100$ $\nabla$ (d) $\nabla=0.7$ and $\nabla=200$ 㽖. (e) Refractive index dependency of spectral peak position. 